\title{
Multiplet effects in Resonant X-ray Emission
}

\author{
Frank M.F. de Groot \\ Department of Inorganic Chemistry and Catalysis, Utrecht University, \\ Sorbonnelaan 16, 3584 CA Utrecht, the Netherlands.
}

\begin{abstract}
After a short discussion of all conventional core level spectroscopies within the single particle model, the effects of the coupling of the core and valence wave function on the $\mathrm{x}$-ray emission spectral shapes is discussed. It will be shown that these so-called multiplet effects strongly affect all $x$-ray emission spectra taken around the metal $2 p$ resonances. In case of $1 \mathrm{~s}$ resonances, valence band $x$-ray emission is not affected, but the spectral shapes of $1 \mathrm{~s} 2 \mathrm{p}$ and $1 \mathrm{~s} 3 \mathrm{p}$ $\mathrm{x}$-ray emission can only be sensibly described with the inclusion of multiplets. A special example is the resonant excitation into the pre-edge region, which gives rise to a quadrupole resonance.
\end{abstract}

\section{CORE LEVEL SPECTROSCOPIES}

All core level spectroscopies will be introduced using a simplified single particle model. This model is not expected to give a correct interpretation of the spectral shapes and intensities observed, but serves as a starting point on which more complete models build upon.

\section{The Single Particle Approximations}

In order to get a first idea of how the x-ray and electron spectra will look like we use a simplified single particle model. A series of approximations have been made:

1. Density-of-States approximation: The assumption that the single particle Density-of-States (DOS), as calculated for example by Local Spin Density (LSD) calculations or by real space Multiple Scattering codes, gives a good account of the electronic structure of the ground state.

2. Spectroscopy approximation: The assumption that the ground state DOS can in fact be used to describe spectroscopic transitions. This implies, for example, that it is assumed that in photoemission the valence hole does not cause a redistribution of the electronic states.

3. Core hole approximation: The assumption that the core hole created in x-ray absorption does not modify the DOS.

4. Matrix Element approximation: The assumption that the transition matrix elements are constant over the energy range analyzed. 
Some of these omitted effects are relatively easy to include. For example, it is in general straightforward to include the matrix element effects. In electronic structure models based on real-space multiple scattering, it is also straightforward to include the core hole. The closely connected approximations (1) and (2) are more difficult to include and are related to electron-correlation effects that are not included in meanfield electronic structure methods.

\section{The Core Hole Spin-Orbit Splitting}

The coupling of the orbital and the spin-moment is given by the spin-orbit interaction, which is essentially a relativistic effect. The spin-orbit interaction is large for core holes and in general two peaks or structures will be visible in the spectrum, separated by the core hole spin-orbit splitting. The relative intensity of the $2 \mathrm{p}_{1 / 2}$ and $2 \mathrm{p}_{3 / 2}$ peak is $1: 2$ (given by the degeneracy of the states) and that of the $3 \mathrm{~d}_{3 / 2}$ to $3 \mathrm{~d}_{5 / 2}$ peak is 2:3. This rule is general for all core level spectroscopies. The rule breaks down only if another interaction is able to mix the $2 \mathrm{p}_{1 / 2}$ and $2 \mathrm{p}_{3 / 2}$ states. It will be discussed below that an important interaction is the overlap of the core hole wave function with the valence state wave functions, or in other words, the coupling of the core and valence moments. This coupling destroys the simple picture sketched above.

\section{X-ray Absorption and X-ray Photoemission}

It has been shown that the x-ray absorption (XAS) process can be described with the dipole approximation, under which the orbital quantum number must be modified by one while the spin quantum number is conserved. This implies that if a 1s-core electron is excited one observes the empty DOS of p-character. This p-projected unoccupied DOS is abbreviated as $\mathrm{C}_{\mathrm{p}}$. Similarly the excitation of a $\mathrm{p}$ core electron probes $C_{s}$ plus $C_{d}$. The Fermi level is reached if the x-ray energy $\hbar \omega$ is exactly equal to the binding energy of the core hole $\left(\mathrm{E}_{1 \mathrm{~s}}\right)$. The unoccupied DOS is reproduced by the spectrum with energies $\mathrm{C}=\hbar \omega+\mathrm{E}_{1 \mathrm{~s}}$, with the $1 \mathrm{~s}$ binding energy given with a negative number.

The same core hole excitation process can excite an electron out of a solid. Its kinetic energy $\left(\mathrm{E}_{\mathrm{k}}\right)$ can be detected and gives directly the binding energy $(\mathrm{Ec})$ of the core electron as: $\mathrm{E} \underline{c}=\mathrm{E}_{\mathrm{k}}-\hbar \omega$, with the binding energy given as a negative number (and omitting the effects of the work function). The 1s XPS spectral shape thus consists of a single line. If a valence electron (V) is excited, exactly the same formula implies: Ec $=E_{k}-\hbar \omega$. An electron at the Fermi level is detected if the kinetic energy of the emitted electron exactly equals the x-ray energy, i.e. the occupied DOS can be determined by subtracting the $x$-ray energy from the kinetic energy: $V=E_{k}-\hbar \omega$, again using negative numbers for the occupied DOS. In principle the dipole selection rule applies again, but because the emitted electron can have any angular momentum in practice the dipole selection rule is dysfunctional and one observes the total occupied DOS (within the imposed approximation on matrix elements). Inverse Photoemission (IPES) is the inverse process of photoemission: an electron is directed towards the sample, it is absorbed in the unoccupied DOS, thereby emitting an x-ray. 


\section{X-ray Emission and Auger}

The core hole decay can take place radiatively by a x-ray emission (XES) process or non-radiatively by an Auger process (AES). XES follows the same selection rules as $\mathrm{x}$-ray absorption and a $1 \mathrm{~s}$-core hole can be filled by a core $2 \mathrm{p}$ electron or a valence $\mathrm{p}$ electron. If a $2 \mathrm{p}$ core electron fills the $1 \mathrm{~s}$ core hole the $\mathrm{x}$-ray energy gives the difference in binding energy of the two core states $E_{2 p}-E_{1 s}=\hbar \omega^{\prime}$. If a valence electron fills the $1 \mathrm{~s}$ core hole one maps the p-projected occupied DOS: $V=\hbar \omega^{\prime}+\mathrm{E}_{1 \mathrm{~s}}$.

Instead of the dipole matrix element $r$, decay of a core hole can occur via the electrostatic two-electron integrals $\langle a b|1 / \mathrm{r}| \mathrm{cd}\rangle$, where $\mathrm{a}, \mathrm{b}, \mathrm{c}$ and $\mathrm{d}$ are electronic states. An example is the matrix element $\left\langle 1 \mathrm{~s} \varepsilon_{\mathrm{d}}|1 / \mathrm{r}| 2 \mathrm{p} 2 \mathrm{p}\right\rangle$. In this Auger process one $2 p$ electron fills the $1 \mathrm{~s}$ core hole and the other $2 \mathrm{p}$ electron is excited out of the solid as a free electron. In this $1 \mathrm{~s} 2 \mathrm{p} 2 \mathrm{p}$ ( or $\mathrm{KL}_{2,3} \mathrm{~L}_{2,3}$ ) Auger process the final state contains two $2 p$-core holes. The kinetic energy of the emitted electron equals $E_{k}=E_{2 p}+E_{2 p}-E_{1 s}$. If a $2 \mathrm{p}$-core electron and a valence electron take part in the Auger process one can detect again the occupied DOS. Neglecting the Auger matrix elements (selection rules), we assume that the total occupied DOS is detected: $V=E_{k}-E_{2 p}+E_{1 s}$. It is also possible that two valence electrons take part in the Auger process. In this 1sVV Auger process one detects the self-convolution of the occupied DOS: $2 \mathrm{~V}=\mathrm{E}_{\mathrm{k}}+\mathrm{E}_{1 \mathrm{~s}}$.

\section{Resonant PES, AES and IPES}

In the foregoing, core hole creation (XAS) has been described separately from core hole decay (XES and AES). However as soon as core hole creation takes place decay occurs. This implies that close to the XAS absorption resonance's, the decay processes can be different from off-resonance excitations. The only effect within the single particle model is that the excited electron can take part in the decay process. This creates additional decay channels not present in off resonant or normal AES and XES.

The best-known resonant spectroscopy is resonant photoemission (R-PES). Within the single particle model this can be described as the two-step process of $\mathrm{x}$-ray absorption followed by Auger, i.e. $\Phi_{0} \rightarrow^{[\mathrm{XAS}]} \rightarrow \underline{1 \mathrm{~s}} \mathrm{C} \rightarrow{ }^{[\mathrm{AES}]} \rightarrow \mathrm{V}$, where $\Phi_{0}$ is the ground state and $\underline{1 \mathrm{~s}}$ a $1 \mathrm{~s}$ core hole. In the final state, a hole exists in the valence band and one measures the occupied DOS as in a normal photoemission process, but a difference is caused by the matrix element. The direct PES channel and the indirect XAS+AES channel have the same initial and final states, hence they interfere with each other.

There is another possible R-PES channel, i.e. $\Phi_{0} \rightarrow^{[\mathrm{XAS}]} \rightarrow \underline{1 \mathrm{~s}} \mathrm{C} \rightarrow \rightarrow^{[\mathrm{AES}]} \rightarrow \mathrm{V}+\mathrm{V}+\mathrm{C}$. In this case the $\mathrm{C}$ electron of the intermediate state does not participate in the Auger decay and this process is called the spectator channel. The process in which the $\mathrm{C}$ does participate is called the participator channel. The final state of the spectator channel has two valence holes (V) plus an extra electron in $\mathrm{C}$. This can be viewed as a normal photoemission final state, plus a $\mathrm{V}$ to $\mathrm{C}$ excitation. This final state cannot be reached by normal PES within the single particle model. It plays a crucial part of all many body descriptions of core level spectroscopy, i.e. it is essentially the shake-up channel. Going to off-resonance conditions, the spectator channel disappears, as the $\mathrm{C}$ electron becomes a free electron. 
Another resonant photoemission process is for example $2 \mathrm{p} 3 \mathrm{pV}$ R-PES, in which first a $2 p$-core hole is created at resonance that subsequently decays by Auger to a $3 p$ core hole, i.e. $\Phi_{0} \rightarrow{ }^{[\mathrm{XAS}]} \rightarrow 2 \underline{\mathrm{pC}} \rightarrow{ }^{[\mathrm{AES}]} \rightarrow \underline{3 \mathrm{p}}, \underline{3 \mathrm{p}}+\mathrm{V}+\mathrm{C}$. The final state in the participator channel is again equal to normal $3 p$ excitation, while the spectator channel adds a $\mathrm{V}$ to $\mathrm{C}$ excitation. Resonant Auger spectroscopy (R-AES) is the same as RPES. The name R-AES will be reserved however for those processes that cannot be reached by direct photoemission. In practice this are all final states with two core holes present in the final states. An example is the 2p3p3p R-AES process, in which first a $2 \mathrm{p}$-core hole is created at resonance that subsequently decays by Auger to two 3p-core holes, i.e. $\Phi_{0} \rightarrow^{[\mathrm{XAS}]} \rightarrow \underline{2 \mathrm{pC}} \rightarrow^{[\mathrm{AES}]} \rightarrow \underline{3 \mathrm{p} 3 \mathrm{pC}}$. Only spectator R-AES processes exist, because if the $\mathrm{C}$ electron participates one never creates a two-hole final state. If one moves away from resonance, the created $\mathrm{C}$ electron becomes a free electron, implying that the R-AES channels disappear.

The reverse process, resonant inverse photoemission (R-IPES), is also possible. If one excites with electrons that have a kinetic energy equal to the binding energy of a core state, an Auger process can occur in which both the core electron and the impinging free electron are transferred to an electron in the conduction band. In a second step, a conduction electron can decay by XES to the core hole. This could again be called a participator channel. In the spectator channel a valence electron decays to the core hole, i.e. $\Phi_{0} \rightarrow^{[\mathrm{AES}]} \rightarrow \underline{2 \mathrm{pCC}} \rightarrow^{[\mathrm{XES}]} \rightarrow \mathrm{C}, \mathrm{C}+\mathrm{C}+\mathrm{V}$.

\section{Resonant XES}

It is also possible to study XES processes at resonance. For example one can excite a $2 \mathrm{p}$ core electron and detect valence band XES. In that case 'participator' R-XES (RXES) equals the resonant elastic scattering path, i.e. $\Phi_{0} \rightarrow^{[\mathrm{XAS}]} \rightarrow 2 \mathrm{pC} \rightarrow \rightarrow^{[\mathrm{XES}]} \rightarrow \Phi_{0}$, $\mathrm{V}+\mathrm{C}$. One observes essentially dd-excitations in a 2p R-XES experiment. Because of its similarity to Raman spectroscopy, R-XES is also called Resonant X-ray Raman Spectroscopy (R-XRS). Yet another name, often used to stress the relation to offresonant inelastic scattering, is Resonant Inelastic X-ray Scattering (R-IXS).

Another R-XES channel is 2 p3s R-XES, i.e. $\Phi_{0} \rightarrow^{[\mathrm{XAS}]} \rightarrow \underline{2 \mathrm{pC}} \rightarrow^{[\mathrm{XES}]} \rightarrow \underline{3 \mathrm{~s} C}$. The final state consists of a $3 \mathrm{~s}$-core hole and an electron in the d-projected unoccupied DOS. The energy difference between the incoming $(\hbar \omega)$ and emitted $\left(\hbar \omega^{\prime}\right) \mathrm{x}$-rays, plus the (negative) $3 \mathrm{~s}$ binding energy, equals the energy of the $\mathrm{C}$ state: $\mathrm{C}=\mathrm{E}_{3 \mathrm{~s}}+\hbar \omega-\hbar \omega$ '. In other words $2 \mathrm{p} 3 \mathrm{~s}$ R-XES is just a complicated detection technique of $2 \mathrm{p}$ XAS (within the single particle model).

Table 1 contains all conventional core level spectroscopies and the results obtained within the single particle approximation. The spectral shape is given by the conservation of energy between initial and final states. For example, 1s XAS is given by $\mathrm{C}-\mathrm{E}_{1 \mathrm{~s}}=\hbar \omega$. Square brackets indicate an alternative experiment. Boldface implies that this energy is varied to measure the spectrum and it can be seen immediately that XAS and XES are x-ray spectroscopies, while PES, IPES and AES are electron spectroscopies. All resonant experiments do contain two variable energy scales. Note that within the single-particle approximation, XAS, IPES, R-AES and R-IPES all have the conduction band structure as there result. The main reasons that in experiment, 
XAS and IPES have different spectral shapes are the differences in matrix elements and the core hole effect.

\begin{tabular}{|c|c|c|c|}
\hline Spec. & Core Holes & Spectral Shapes & DOS \\
\hline XAS & $1 \mathrm{~s}$ & $C-E_{1 s}=\hbar \omega$ & $\mathrm{C}$ \\
\hline XPS & $2 \mathrm{p}[\mathrm{V}]$ & $E_{2 p}[V]=\mathbf{E}_{\mathbf{k}}-\hbar \omega$ & {$[\mathrm{V}]$} \\
\hline IPES & - & $\mathrm{C}=\mathbf{E}_{\mathbf{k}}-\hbar \omega$ & $\mathrm{C}$ \\
\hline XES & $1 \mathrm{~s} 2 \mathrm{p} \quad[1 \mathrm{sV}]$ & $\mathrm{E}_{2 \mathrm{p}}-\mathrm{E}_{1 \mathrm{~s}} \quad\left[\mathrm{~V}-\mathrm{E}_{1 \mathrm{~s}}\right]=\hbar \omega^{\prime}$ & $-\quad[\mathrm{V}]$ \\
\hline AES & $1 \mathrm{~s} 2 \mathrm{p} 2 \mathrm{p} \quad[1 \mathrm{sVV}]$ & $2 \mathrm{E}_{2 \mathrm{p}}-\mathrm{E}_{1 \mathrm{~s}}\left[2 \mathrm{~V}-\mathrm{E}_{1 \mathrm{~s}}\right]=\mathbf{E}_{\mathbf{k}}$ & $-\quad[2 \mathrm{~V}]$ \\
\hline R-PES & $1 \mathrm{sVV} \quad[1 \mathrm{sVC}]$ & $\mathrm{V}[2 \mathrm{~V}-\mathrm{C}]=\mathbf{E}_{\mathbf{k}}-\hbar \omega$ & $\mathrm{V} \quad[2 \mathrm{~V}-\mathrm{C}]$ \\
\hline R-AES & $2 p 3 p 3 p$ & $2 \mathrm{E}_{3 \mathrm{p}}-\mathrm{C}=\mathbf{E}_{\mathbf{k}}-\hbar \omega$ & $\mathrm{C}$ \\
\hline R-XES & $2 \mathrm{p} 3 \mathrm{~s} \quad[2 \mathrm{pV}]$ & $\mathrm{C}^{-\mathrm{E}_{3 \mathrm{~s}}}[\mathrm{C}-\mathrm{V}]=\hbar \omega-\hbar \omega^{\prime}$ & $\mathrm{C} \quad[\mathrm{C}-\mathrm{V}]$ \\
\hline R-IPES & $2 \mathrm{pC} \quad[2 \mathrm{pV}]$ & $\mathrm{C}[2 \mathrm{C}-\mathrm{V}]=\mathbf{E}_{\mathbf{k}}-\hbar \boldsymbol{\omega}$ & {$[2 \mathrm{C}-\mathrm{V}]$} \\
\hline
\end{tabular}

\section{BEYOND THE SINGLE PARTICLE MODEL}

The single particle interpretation gives a good overview of the potential use of the various spectroscopies. One of the important applications of resonant experiments is in fact the determination of the limitations of the single particle models, or in other words, the study of many body effects. The many body effects related to the ground state electronic structure will not be discussed in this paper. I will focus only on another set of important interactions not included in the single particle model and being crucial in all states containing a core hole. This is the overlap of the core wave function and the valence wave function. The effects of this interaction are known as multiplet effects. In the remainder of this paper, the consequences of multiplet effects on XAS and resonant XES will be discussed. If we limit ourselves to core levels of the $3 d$-metals, multiplet effects are important in all cases where a $2 p, 3 p$ or 3 s hole is present. In addition, the $3 \mathrm{~d}$-valence band is susceptible to strong correlation effects, but these will not be discussed in this paper. We consider the following cases:

- 2 p XAS: multiplet effects in final state ${ }^{[1,2,3]}$

- 2p3s R-XES: multiplet effects in intermediate states and interference effects ${ }^{[4,5]}$

- 2p3d R-XES: dd-excitations and spin-flip excitations ${ }^{[6,7,8]}$

- $1 \mathrm{sV}$ XES: a probe of the valence band $\operatorname{DOS}^{[9]}$

- 1s2p R-XES: multiplet effects in the intermediate and final states ${ }^{[10]}$

\section{2p x-ray absorption}

In case of $2 \mathrm{p} x$-ray absorption, the single particle model assumes the transition from the ground state to $2 \mathrm{pC}$. The overlap of the $2 \mathrm{p}$ and $3 \mathrm{~d}$ wave function is very strong and gives rise to energy effects of the order of $10 \mathrm{eV}^{1,2}$. The spectral shape is completely dominated by the multiplet effects and any relation to a single particle interpretation is lost. For example, the $2 \mathrm{p} 3 \mathrm{~d}$ overlap makes the $\mathrm{MnO}$ spectrum look closely like the $2 \mathrm{p}^{5} 3 \mathrm{~d}^{6}$ final state multiplet, implying that the ground state of $\mathrm{MnO}$ is dominated by the $3 \mathrm{~d}^{5}$ configuration $^{[3]}$. The multiplet effects can be viewed as a kind of 'energy microscope' that enlarges small initial state energy effects to much larger final state 
energy effects. As such meV energy differences can be made visible with $100 \mathrm{meV}$ resolution $^{[3]}$.

\section{2p3s resonant $x$-ray emission}

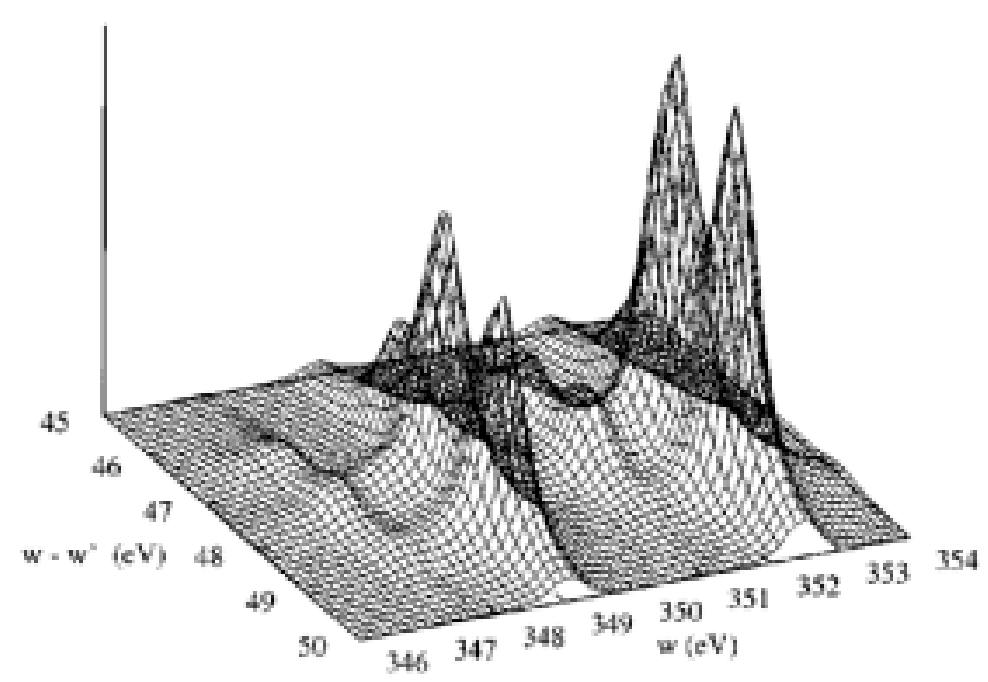

FIGURE 1. Intensity plot of the RIXS cross sections, calculated using Eq. 1, that is including interference effects. Excitation energy $(\omega)$ on the $x$ axis; final-state energy $\left(\omega-\omega^{\prime}\right)$ on the $y$ axis. [reproduced from reference 5].

In case of 2p3s R-XES, the intermediate state is strongly affected by multiplet effects. In general a large number of intermediate states are present, which can give rise to complicated interference effects because the life time broadening is equivalent in size to the splitting between the levels. In the next section we discuss the complex case of $\mathrm{Mn}$, but here we focus on the simplest case, being that of a system with an empty $3 \mathrm{~d}$ band in the ground state, such as $\mathrm{Ca}^{2+}$ compounds as $\mathrm{CaF}_{2}$. The ground state has a $3 \mathrm{~d}^{0}$ configuration, implying a final state of $2 p$ x-ray absorption with a $2 p^{5} 3 d^{1}$ configuration. The transitions can be essentially calculated by hand. It has been shown that this 2p x-ray absorption spectrum consists of seven final states, which are well separated in the experiment ${ }^{[1]}$. On each of these seven peaks one can study the resonant $2 \mathrm{p} 3 \mathrm{~s}$ x-ray emission decay. The experimental results ${ }^{[4]}$ have been simulated with multiplet calculations, using the Kramers-Heisenberg formula:

$$
I\left(\omega, \omega^{\prime}\right) \sim \sum_{f}\left|\sum_{x} \frac{\left\langle\Phi_{f}\left|\hat{e}^{\prime} \cdot r\right| \Phi_{x}\right\rangle\left\langle\Phi_{x}|\hat{e} \cdot r| \Phi_{i}\right\rangle}{\omega-E_{x}-i \Gamma_{x}}\right|^{2} \delta_{E_{f}+\hbar \omega^{\prime}-E_{i}-\hbar \omega}
$$

with $\omega$ being the incoming x-rays, $\omega^{\prime}$ the emitted x-rays, $\Phi_{\mathrm{I}}$ the initial state, $\Phi_{\mathrm{x}}$ the intermediate state and $\Phi_{\mathrm{f}}$ the final states. (See ref. ${ }^{[5]}$ for details). The resonance part of the experiments can be nicely simulated with these calculations. The simulations can be represented as a two-dimensional plot of $\omega$ and $\omega^{\prime}$ as given in Figure 1. In practice one uses the energy of the final states, being equal to the difference between $\omega$ and $\omega$ '. The final state energy gives the binding energy of the $3 \mathrm{~s}^{1} 3 \mathrm{~d}^{1}$ final states. The only 
multiplet effect active in this $3 s^{1} 3 d^{1}$ configuration is the $3 s 3 d$ exchange interaction. The only other energy effect (within the model used) is the cubic crystal field, and four peaks are found split by these two interactions.

\section{2p3d resonant $x$-ray emission}

The first 2p3d R-XES study has been carried out by Butorin and coworkers ${ }^{[6]}$. They studied the $2 \mathrm{p} 3 \mathrm{~d}$ decay at the $2 \mathrm{p}$ x-ray absorption resonances of $\mathrm{MnO}$. The experiments show a series of peaks at energy losses $\left(\omega-\omega^{\prime}\right)$ equal to the well known dd-excitations. The general trends of the data could already be explained from an atomic multiplet model ${ }^{[6]}$. The details of the dd-transitions and the charge transfer peaks can be explained by using the more complete charge transfer multiplet model $^{[3,7]}$. By using this model, the ff-transitions in rare earth $3 \mathrm{~d} 4 \mathrm{f}$ R-XES spectra have been made visible. Details on these experiments can be found in a recent review article on RIXS ${ }^{[8]}$.

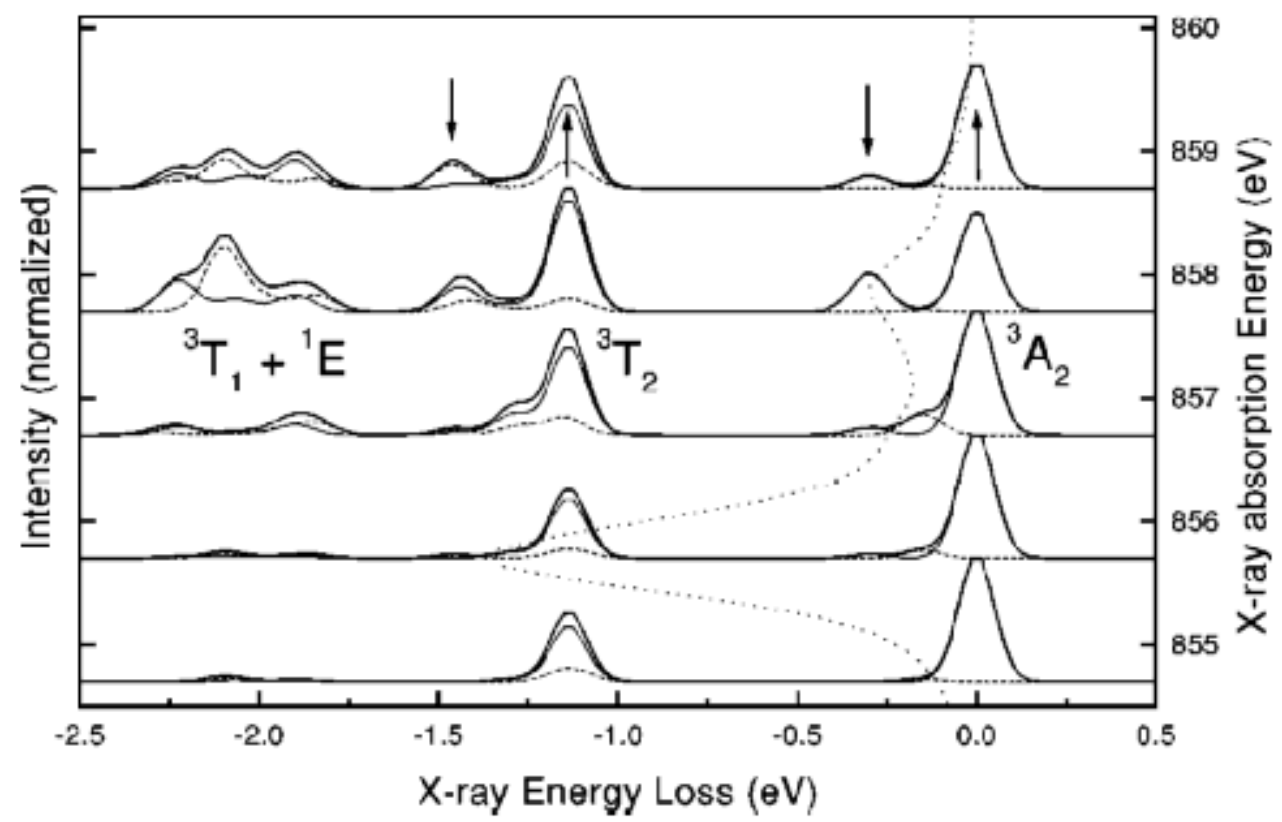

FIGURE 2. $2 \mathrm{p} 3 \mathrm{~d}$ RIXS at the $2 p$ edge of $\mathrm{N} \mathrm{Ni}^{2+}$ system in octahedral symmetry, using the crystal-field parameter of $\mathrm{NiO}$. Indicated are $F_{\mathrm{xx}}$ scattering (thin solid), $F_{\mathrm{zx}}$ scattering (dashed), and the total scattering (thick solid). The x-ray-absorption spectrum is given with dots. The symmetries of the states are given at the middle spectrum. The $M_{\mathrm{s}}$ sub-states are indicated at the top for spin up and spin down (figure reproduced from Reference 7).

In this paper, I would like to focus on yet another phenomenon of $2 p 3 d$ R-XES, the possibility to observe spin-flip transitions. The usage of the term spin-flip is not consistent in articles on R-XES/R-XRS/RIXS, so I will try to clarify the different usage's here. Sometimes part the dd-transitions are called spin-flip transitions because the total spin quantum number $\mathrm{S}$ of the configurations is changed from, for example, $5 / 2$ to $3 / 2$ as is the case for the $\mathrm{MnO}$ experiments. Instead of a change in $\mathrm{S}$, also a change in $M_{S}$ is called spin-flip. I will consider a change in $M_{S}$ as a real spin-flip 
transition, the change in $\mathrm{S}$ being called a multiplet excitation, as it are the multiplet effects that cause the energies involved. Multiplet excitations are of the order of a few $\mathrm{eV}$, whereas spin-flip excitations are of the order of $100 \mathrm{meV}$, being closely related to the superexchange energy ${ }^{[7,9]}$. With the improvement in the resolution of soft $\mathrm{X}$-ray $\mathrm{R}$ XES experiments, these $0.1 \mathrm{eV}$ transitions can be made visible in experiment ${ }^{[9]}$.

\section{1sV x-ray emission}

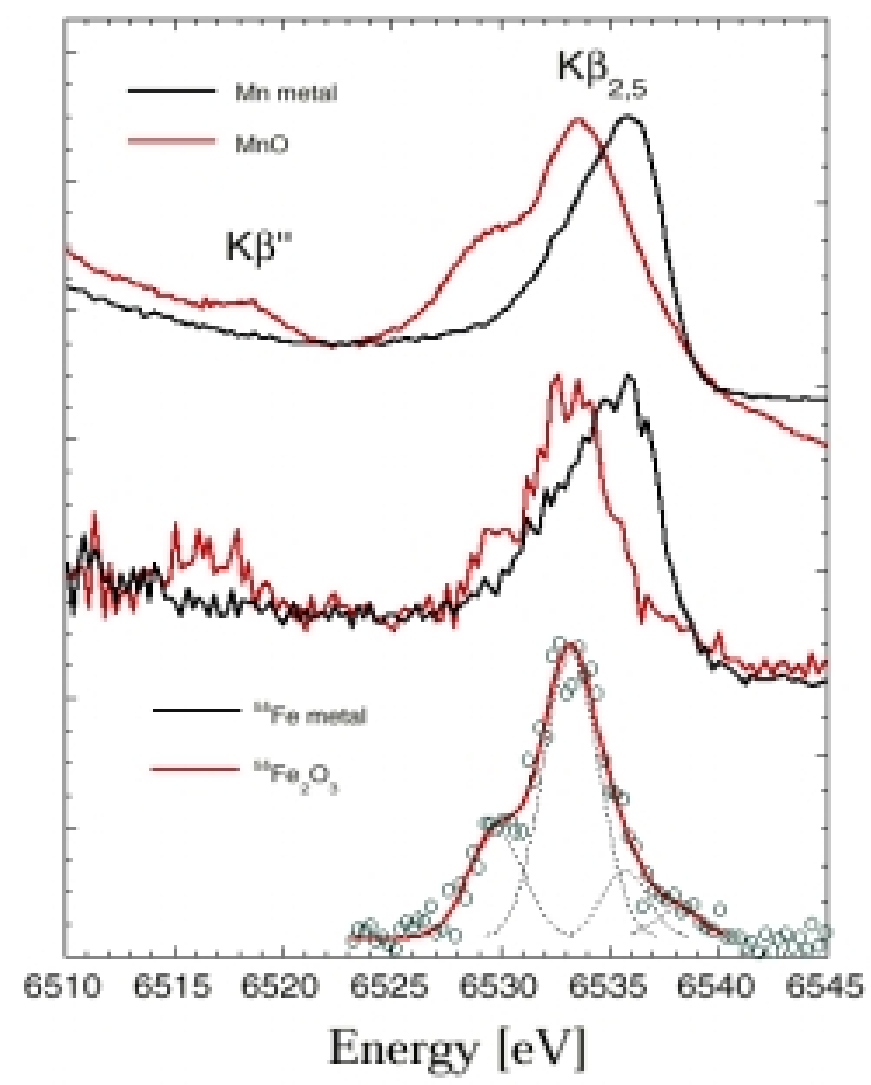

FIGURE 3. Valence band $1 \mathrm{sV}$ x-ray emission spectra. Top to bottom: (a) X-ray excited spectra for $\mathrm{MnO}$ (red) and $\mathrm{Mn}$ metal (black); (b) K-capture spectra for $55 \mathrm{Fe}_{2} \mathrm{O}_{3}$ (red) and Fe metal (black); (c) a fit of the background-subtracted valence band region. (reproduced from reference [10]).

In case of $1 \mathrm{~s} x$-ray absorption, the main edge is due to $1 \mathrm{~s}$ to $4 \mathrm{p}$ transitions. Its spectral shape is hardly affected by multiplet effects. The situation is different for the pre-edges that are discussed in the next section. Valence band emission to the 1s core hole, on the main edge as well as off-resonance, leaves the final state with a valence hole and an essentially free electron that can be neglected in the description of the $1 \mathrm{sV}$ $\mathrm{x}$-ray emission spectral shape. Thus, $1 \mathrm{sV}$ x-ray emission is not affected by multiplets and describes the valence band DOS, similar to valence band XPS. A difference is caused by the matrix elements that in case of $1 \mathrm{sV}$ x-ray emission allow transitions only from the p-projected DOS. In case of 3d-metal oxides, this implies the metal pstates in the oxygen $2 p$-valence band. In addition at threshold, weak quadrupole transitions from the occupied 3d-states can be expected, in a way similar to the preedge structures in $1 \mathrm{~s}$ XAS, i.e. including the admixture effects of $4 \mathrm{p}$ and $3 \mathrm{~d}$ character. 
Figure 3 shows the valence band region $\left(\mathrm{K} \beta_{2,5}\right.$ in spectroscopic notation). The bottom spectrum shows the oxygen $2 \mathrm{p}$ valence band at 6530 and $6534 \mathrm{eV}$. The intensity around $6538 \mathrm{eV}$ is expected to be due to the $3 \mathrm{~d}$ valence band. The $\mathrm{K} \beta$ " peak at $6515 \mathrm{eV}$ is due to manganese $4 \mathrm{p}$-character in the oxygen $2 \mathrm{~s}$ states. This peak is denoted as a cross-over peak as it can be viewed as a x-ray emission channel from oxygen to manganese. The top spectra excited with $\mathrm{x}$-rays several tens of $\mathrm{eV}$ above the resonance. The middle spectrum shows x-ray emission spectra, where the core hole has been created by the $\mathrm{K}$ capture process. In $\mathrm{K}$ capture ${ }^{55} \mathrm{Fe}$ decays to ${ }^{54} \mathrm{Mn}$ by reaction of a 1s core electron with a proton to a neutron and neutrino. This creates a $\mathrm{Mn}$ atom with a 1s core hole, that subsequently decays similar to an X-ray excited $\mathrm{Mn}$ atom. For more details, including a discussion of the differences in x-ray emission spectral shapes between $\mathrm{K}$ capture and $\mathrm{x}$-ray excited core holes, is referred to ref. ${ }^{[10] .}$

\section{1s2p resonant $x$-ray emission}

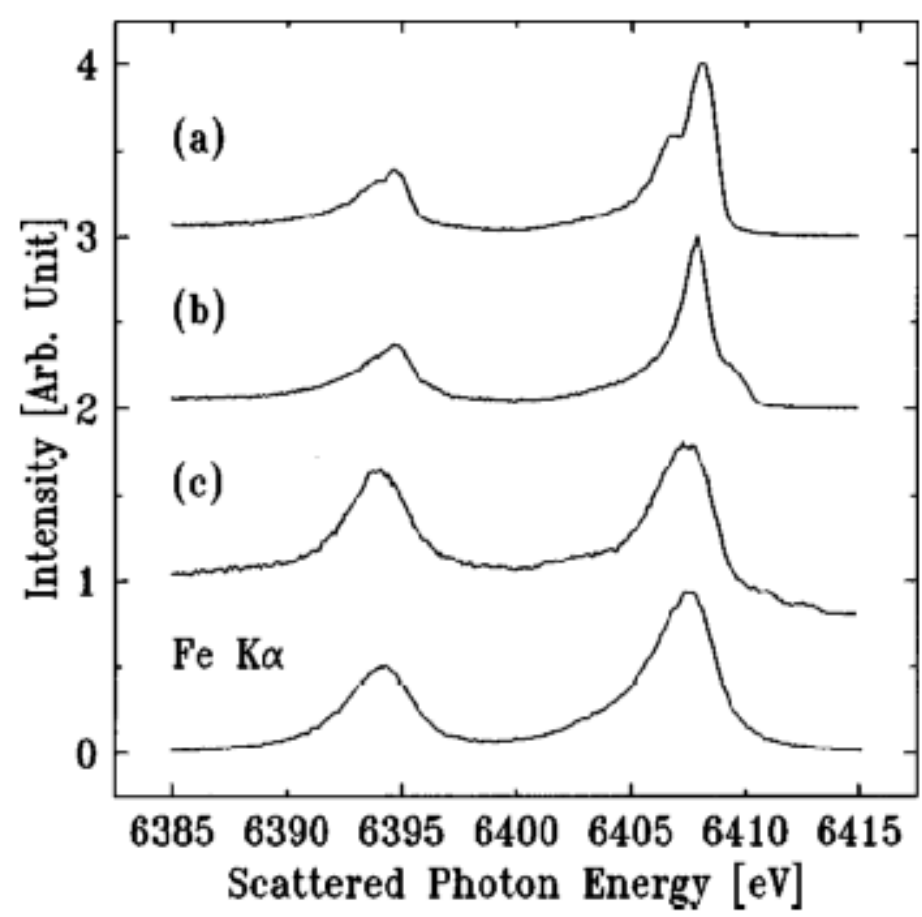

Figure 4: RIXS spectra as a function of the scattered photon energy for three different excitation energies together with the off resonance $1 \mathrm{~s} 2 \mathrm{p}$ x-ray emission spectrum. The excitation energies are 7113.4 (a), 7114.7 (b) and $7117.7 \mathrm{eV}$ (c) for the RIXS spectra (reproduced from Ref. [11]).

As a last example, I would like to discuss the 1s2p x-ray emission spectra, taken at an excitation energy set exactly at the two peaks of the pre-edge structure of the $1 \mathrm{~s}$ XAS spectrum of $\mathrm{Fe}_{2} \mathrm{O}_{3}{ }^{[11]}$. This pre-edge structure consists of two peaks at $7113.4 \mathrm{eV}$ and at $7114.7 \mathrm{eV}$, split by the crystal field splitting of $1.3 \mathrm{eV}$. Both pre-edge peaks have an intensity of a few percent of the main edge and are related to $1 \mathrm{~s} 3 \mathrm{~d}$ quadrupole transitions. This quadrupole excitation is followed by a dipolar $1 \mathrm{~s} 2 \mathrm{p}$ decay. The overall process can be accurately described with the multiplet model for the process 
$3 d^{5} \rightarrow 1 s^{1} 3 d^{6} \rightarrow 2 p^{5} 3 d^{6}$. Note that the final state has exactly the same configuration as would be the case for direct $2 p$ x-ray absorption. Detailed analysis learns that this analogy is indeed correct and that the final state of this process are identical to that of $2 \mathrm{p}$ XAS, the only difference being different transition matrix elements ${ }^{[11]}$.

\section{CONCLUDING REMARKS}

The examples given indicate that the $\mathrm{x}$-ray emission processes that concern a $2 \mathrm{p}, 3 \mathrm{~s}$ or $3 p$ core level in the intermediate or final state are in all cases strongly affected by multiplet effects. In fact this concerns all resonant x-ray emission processes, except off-resonance 1sV XES. Multiplet analysis does yield an accurate simulation of all spectral shapes, and additionally provides valuable insights into the electronic structure of the materials studied (not discussed in this paper).

A very important usage of these R-XES processes is the measurement of XAS spectra by putting the detector at a particular final state. It has been $\operatorname{shown}^{[10]}$ that a wide range of selective XAS spectra can be measured. This includes selectivity's for the valence, the spin-state and the type of neighbour ${ }^{[10,12]}$.

As a last remark, I would like to mention that two recent review paper have appeared on soft X-ray resonant $\mathrm{XES}^{[8,13]}$. A paper by Butorin focuses on the multiplet analysis of transition metal and rare earth spectra ${ }^{[8]}$. A very extensive paper by Gel'mukhanov and Agren ${ }^{[13]}$ can be considered 'orthogonal' to the present contribution as it concerns essentially all spectra for which multiplet effects are not important.

\section{REFERENCES}

1. de Groot, F.M.F, Fuggle, J.C, Thole, B.T. and Sawatzky, G.A., Phys. Rev. B. 41, 928 (1990)

2. de Groot, F.M.F, Fuggle, J.C, Thole, B.T. and Sawatzky, G.A., Phys. Rev. B. 42, 5458 (1990)

3. de Groot, F.M.F. J. Elec. Spec, 67, 529 (1994)

4. Rubensson, J.E., Eisebitt, S., Nicodemus, M., Böske, T, and Eberhardt, W., Phys. Rev. B 49, 1507 (1994)

5 de Groot, F.M.F., Phys. Rev. B. 53, 7099.

6 Butorin, S.M., Guo, J., Magnuson, M. Kuiper, P and Nordgren, J., Phys. Rev. B. 54, 4405 (1996).

7 de Groot F.M.F, Kuiper, P and Sawatzky, G.A. Phys. Rev. B. 57, 14584 (1998)

8 Butorin, S.M., J. Elec. Spec. (in press).

9 Kuiper, P, Guo, J., Såthe, C., Duda, L.C., Nordgren, J., Pothuizen, J.J.M., de Groot, F.M.F. and Sawatzky, G.A., Phys. Rev. Lett. 80, 5204 (1998).

10 Bergmann U, Glatzel P, Degroot F.M.F. and Cramer S.P., J. Am. Chem. Soc. 121, 4926 (1999).

11 Caliebe, W.A., Kao, C.C., Hastings, J.B., Taguchi, M., Kotani, A. Uozumi, T., and de Groot, F.M.F., Phys. Rev. B. 58, 13452 (1998).

12 de Groot, F.M.F., Topic in Catalysis (in press).

13 Gel'mukhanov, F. and Agren, H., Physics Reports 312, 87-330 (1999). 